Original article

\title{
What to expect from high throughput genomics in metastatic breast cancers?
}

\author{
Concetta Elisa Onesti a, b, Cécile Vicier a , Fabrice André a, c, * \\ a Institut National de la Santé et de la Recherche Médicale (INSERM) U981, Institut de cancérologie Gustave Roussy, Villejuif, F-94800, France \\ b Department of Medical Oncology, S. Andrea Hospital, University of Rome "Sapienza », Rome, Italy \\ ${ }^{c}$ Department of Medical Oncology, Institut de cancérologie Gustave Roussy, Villejuif, F-94800, France
}

\section{A R T I C L E I N F O}

\section{Article history:}

Available online 1 August 2015

\section{Keywords:}

High throughput genomics

Next generation sequencing

Comparative genomic hybridization

Metastatic breast cancer

\begin{abstract}
A B S T R A C T
Breast cancer is a heterogeneous disease and its genomic characteristics have been widely studied in the last years. Although several progresses have been made, metastatic disease is still incurable in the majority of patients. Recent genomic studies have shown that a large number of candidate targets exist in breast cancer. Currently only two drivers have been validated (ER and HER2), but several others seem to be associated with objective response, such as PIK3CA mutations, FGFR1 amplifications, AKT1 mutations, EGFR amplifications and ERBB2 mutations. Beside driver identification, many other applications can be developed for genomics such as identification of lethal subclones, DNA repair defects or immune response against tumor. Most of the precision medicine programs currently use targeted sequencing. Nevertheless, whole exome sequencing, RNA sequencing, gene expression analysis, phosphoprotein detection, SNP arrays and ctDNA sequencing have been also proposed in clinical trials.
\end{abstract}

๑) 2015 Elsevier Ltd. All rights reserved.

\section{Introduction}

Numerous models to explain cancer complexity have been proposed up to now, such as that based on the principle of progressive accumulation of DNA mutations, responsible for initiation, progression, dissemination, response and resistance to treatment $[1,2]$. Somatic mutations are abundant in every cancer cells, but most of them do not play a role in cancer progression. They are classified in "passengers", that have not a functional effect but are associated with a clonal expansion; "drivers", associated with a selective advantage; "actionables", that have diagnostic, prognostic or therapeutic significance; and "druggables", that are targets of therapeutic development $[3,4]$. In this context, multigene diagnostic assays, such as next generation sequencing (NGS) technologies or comparative genomic hybridization arrays (CGH), assume primary importance in cancer treatment development [5-7]. Thus genomic technologies are relevant and their applications are growing. Targeting oncogenic drivers can be a possible approach to improve outcome in patient's refractory to standard treatments. Therefore, new clinical trials are required to translate scientific

\footnotetext{
* Corresponding author. Inserm U981, Institut Gustave Roussy, 114 rue Edouard Vaillant, 94805, Villejuif, France. Tel.: +33 (1) 421142 93; fax: +33 (1) 42115217.

E-mail address: fabrice.andre@gustaveroussy.fr (F. André).
}

innovations to clinical practice for patients selected according to their molecular characteristics [8].

Breast cancer is the leading cause of cancer related death worldwide, with about 232.340 new cases of invasive breast cancers and 390.620 deaths in the USA in 2013 [9]. Although in the last years advances have been made in breast cancer management, metastatic disease is still incurable. The majority of progress has been done for targeted therapies, for instance, therapies targeting HER2 with pertuzumab or trastuzumab-emtansine $[10,11]$. However, studies on molecular characterization have shown that breast cancers present many other genomic alterations, including AKT1, PIK3CA and FGFR1, which can be targeted by new molecular drugs $[6,12,13]$.

In this paper we will analyze different applications of high throughput genomic assays in metastatic breast cancers and their involvement in therapeutic development of several targeted agents.

\section{Genomic tests to decipher cancer biology at the individual level}

\section{Oncogenic drivers}

Identification of drivers at the DNA level.

In the last years the concept of precision medicine has been growing. The introduction of high throughput technologies, such as 
whole exome sequencing and copy number analyses has allowed to identify tumor molecular characteristics. The introduction of these techniques advantaged the discovery and update of oncogenic drivers. Targeting drivers gene mutations can lead to oncogene deaddiction and tumor shrinkage. The SAFIR-01 trial has been conducted in this context: guide patients towards specific targeted therapies according to their genetic characteristics [6].

Over the past few years, many other trials have emerged to study genomic characteristics in breast cancer patients, with the identification of about 10-20 candidate actionable drivers, most of them occurring in less than $10 \%$ of patients [12-16]. Many clinical trials have been focused on these new targets, but nowadays only few of them are associated with objective response in clinical studies. Among these we can mention PIK3CA mutations, observed most commonly in hormone receptor positive breast cancers (34,5\%) respect to HER2 positive $(22,7 \%)$ or basal-like tumor $(8,3 \%)$ [17]. In this setting of patients, specific treatment with BYL719, a selective PI $3 \mathrm{~K} \alpha$ inhibitor, in combination with endocrine therapy (letrozole or exemestane) has shown promising results in a phase I study, associated with manageable adverse events [18]. FGFR1 amplifications, present in about $10 \%$ of breast cancer patients and associated with a poor prognosis, showed encouraging results in preclinical studies. There are nowadays several ongoing clinical trials with different compounds, such as lucitanib, dovitinib, AZD4547, nintedanib, BGJ398 and JNJ-42756493 [19-21]. Other rarer gene alterations in breast cancer, such as AKT1 and ERBB2 mutation and EGFR amplification, are present. Mutations of AKT1 occur in about $4 \%$ of breast cancers. Targeting mTOR pathway with specific inhibitors, such as everolimus, leads to promising results in clinical trials [6,22]. ERBB2 mutation is an uncommon alternative mechanism to activate HER2 in breast cancer and is currently considered as a possible target of the HER2 inhibitor neratinib [23]. Finally, EGFR amplification, observed in $2 \%$ of the cases, can be the target of specific EGFR inhibitors [6]. Unfortunately, other identified gene alterations have not shown encouraging results in clinical studies. For example, the amplification of CCND1 is not associated with a better response in patients treated with palbociclib, a cyclindependent kinases 4 and 6 inhibitor, compared to unselected patients. Nevertheless this targeted therapy can improve progression free survival in advanced breast cancer, independently of the CCND1 abnormality, explaining why a phase III trial is ongoing (NCT01942135, www.clinicaltrials.gov) [24]. The presence of coexisting mutations in the same tumor can be associated with resistance to therapy. Hortobagyi and colleagues observed a better response in patients with a mutation activating mTOR pathway, such as PIK3CA, FGFR1 and CCND1, compared to patients not carrying gene mutations. Also a reduction of everolimus efficacy in patients carrying multiple mutations was detected [25].

In summary, in advanced breast cancers, five gene alterations have been associated with objective response: PI3KCA mutations, FGFR1 amplifications, AKT1 mutations, EGFR amplifications and ERBB2 mutations. Five to ten other genes are candidate drivers based on preclinical studies.

Currently, the preferred approach for personalized medicine is to use targeted sequencing and copy number analyses in order to deliver the best treatment.

Targeting the pathways: identification of driver at the RNA/protein level

While most of the current strategies of personalized medicine aim to identify DNA alterations, other approaches could identify pathway activation and dependency. PI3K/mTOR pathway activation is involved in resistance to hormonotherapy in ER-positive breast cancer. The BOLERO-2 trial results highlight that targeting mTOR pathway is an effective strategy to overcome resistance to a previous endocrine therapy [26]. Loi and colleagues identified that mTOR activation was associated with better response with mTOR inhibitor treatment $[27,28]$. Gene expression could be useful to identify drivers of cancer progression and pathway dependency.

Another pathway driving cancer progression is the CDK4/RB pathway, associated with cell cycle progression, through transcription activation of E2F-regulated genes. Abnormal RB pathway is reported to be aberrant in about $20-35 \%$ of breast cancers and is associated with poor outcome. It responds better to cytotoxic drugs than to tamoxifen, due to the bypass of antiestrogen signaling. Therefore, RB pathway can be studied as a biomarker to address the therapeutic choice in ER-positive breast cancers [29].

$\mathrm{ER}, \mathrm{mTOR}$ and CDK4 pathways drive cancer progression in a majority of ER+/Her2- BC. Thus, there is a need to develop molecular tools to assess pathway activation and dependency by gene expression arrays and phosphoprotein arrays.

\section{Lethal subclones and intratumor heterogeneity}

Profiling tumors emphasizes that genetic differences exist not only between different tumors, but also in the same tumor. This intra-tumor heterogeneity is related to genomic instability, causing an additional challenge for developing drugs in presence of different mutations $[30,31]$. Within the tumor multiple differences at genetic and epigenetic levels exist, resulting in therapy resistance. Indeed, Landau and colleagues worked on chronic lymphocytic leukemias and have shown different responses to treatment based on DNA methylation tumor profile at an epigenetic level [32]. However genomic instability results in dynamic alterations in clonal and subclonal frequencies. Selective pressure, determined by treatment and tumor microenvironment, leads to a selection of specific subclones responsible of resistance to target therapy [33]. Lethal subclones can be present in a minority of cells in primary tumor. Ultradeep sequencing could allow early identification and provide the information for specific treatment [34]. Several studies have shown that targets can be lost or gained during tumor evolution, putting in evidence the need of a new biopsy at the time of disease progression [35]. Currently the re-biopsy is not a clinical practice due to the invasiveness of the procedure. Recent studies have shown that genomic changes occurring during the course of the disease can be detected with liquid biopsy, by sequencing circulating DNA at baseline and at disease progression. Circulating DNA could monitor the appearance of lethal clones, without invasive procedures [36].

Several gene alterations such as ESR1, TSC1/2 and PTEN have been associated with resistance genotype. To illustrate, Arnedos et al. observed that metastatic breast cancers in comparison with the primary tumors present higher level of genes involved in migration processes or in resistance mechanisms, such as ESR1 or TSC1 [37]. Toy and colleagues observed that the ESR1 was frequently mutated at metastatic site, but not in primary tumor samples. Overall the mutation was detected in less than $1 \%$ of early breast cancer and in about $20 \%$ of aromatase inhibitor resistant tumors and is associated with a poor prognosis. They identified that the mutation involves the ligand binding domain of ESR1, which is responsible of acquired resistance to endocrine therapy through the activation independent to the ligand. However, drugs directly targeting ER and inducing its proteasomal degradation, such as fulvestrant, may be effective against the mutant forms of estrogen receptor [38,39]. Moreover, PTEN mutations and deletions could be a mechanism of resistance to PI3K inhibitors. Indeed, the progressive decrease or loss of PTEN during treatment with BYL719 might lead to PI3K/AKT pathway activation and consequently to tumor growth [40]. 
In conclusion, considering that some genes are associated with resistant disease, identifying these lethal minor subclones by ultradeep sequencing and ctDNA could be useful.

\section{Mutagenesis processes and DNA repair defects}

Somatic mutations in the cancer genome could be a consequence of DNA replication error, exposure to mutagen or defective DNA repair mechanisms. DNA repair defects are responsible of cancer progression, through accumulation of multiple DNA alterations. Less than $5 \%$ of breast cancers present mutations of BRCA 1 and BRCA2 genes, correlated to hereditary breast cancer with $80 \%$ of lifetime risk when one of the genes is mutated [41-43]. BRCA1 and 2 proteins are involved in double strand break (DBS) repair mechanisms and their mutations are responsible of homologous recombination deficiency (HRD). In BRCA1 or BRCA2 tumors, poly (ADP-ribose) polymerase (PARP) inhibitors can lead to tumor regression by a process known as synthetic lethality, a result of the accumulation of un-repaired DNA DSB and an unsupportable increase in genomic instability. The loss of PARP activity results in an accumulation of new DNA damages, normally repaired by HR mechanism, with consequent induction of cell death [44]. Acting on synthetic lethality with DNA repair defect (PARP inhibitors and BRCA defects) represents new targets on mutational process. Genomic tests have been proposed to identify defects in DNA repair pathways and the best model is with the BRCA genes. To illustrate, a phase II clinical trial with olaparib, a PARP inhibitor has shown promising results in metastatic breast cancer. 54 patients were enrolled in two cohorts; the ones treated with $400 \mathrm{mg}$ twice daily have shown the majority objective response and stable disease [45]. Currently a phase III trial is ongoing (NCT02000622, www. clinicaltrials.gov). In the same field, an ongoing phase II study ARIEL2 (NCT01891344, www.clinicaltrials.gov), is recruiting patients with platinum sensitive ovarian cancer, BRCA mutated or wild type. Rucaparib, another PARP inhibitor, is administered. In preliminary results, this study identified an HRD signature, characterized by high tumor genomic loss of heterozygosis (LOH). This signature could be present in both BRCA wild type and mutated patients and is associated with a clinical benefit. In this trial HRD status is assessed using NGS technique [46]. Furthermore, another application of whole exome sequencing is to predict which DNA repair defect is altered on the basis of the mutational signature detected in each individual [47].

In conclusion, to analyze DNA repair defects different techniques are now available: the single gene sequencing, for example for BRCA1 and BRCA2 genes; the whole exome sequencing, to detect a HRD signature predicting response to PARP inhibition or to identify a mutational signature suggesting a specific DNA repair defect; and the SNP arrays to assess HRD status, through detection of $\mathrm{LOH}$.

\section{Dialogue between cancer cells and immune system}

Immunotherapy has led to outcome improvement in different cancers, especially in melanoma. Several studies correlated immunotherapy outcomes to peripheral-blood lymphocyte count, tumor microenvironment, and maintenance of high-frequency Tcell receptor clonotypes [48-50]. Also in connection with new tools and technology, potential applications of genomics could be to identify immune defects in individuals with metastatic breast cancer. Interestingly, Snyder and colleagues have shown in melanomas that a high mutational load has enabled to improve outcomes with anti-CTLA-4 treatment. The authors has supplemented on the fact that a high load alone is not sufficient to predict the efficacy. In fact, certain cancers are highly mutated, but do not respond to immunotherapy. Moreover, the presence of neoantigens in the tumor could be involved in treatment response. This neoantigens burden, recognized by host immune system, appears to be determinant for immunotherapy efficacy [51]. Furthermore, cancer cells could release signal linked to cell death in order to trigger immune response. Genetic analysis could evaluate whether the host generate an immune response following cancer cells death. For example, in breast cancer the detection of toll-like receptor 4 (TLR4) loss of function is related to a rapid relapse following radiotherapy or chemotherapy [52]. Finally, metastatic site biopsies analysis have shown a very low level of TILs, PD1 and PDL1, except for HER2 positive metastatic breast cancers, suggesting the presence of an immune suppressive network in this stage of the disease [37].

\section{Conclusion}

Breast cancer is a complex disease with a progressive genetic evolution, responsible of different molecular characteristics in each stage of the disease and of the acquired resistance to target therapies. Only few targets are currently validated in breast cancer treatment, such as ER and HER2, but several other oncogenic drivers are being studied with encouraging results. In recent year, the concept of personalized medicine has grown and the high throughput genomic analysis became fundamental for treatment decisions. Different techniques are used for genomic analysis and they depend on what clinicians want to target in metastatic breast cancer. NGS is used to detect oncogenic drivers (ERBB2 amplification or mutations, PIK3CA mutations, AKT1 mutations). Gene expression or phosphoproteine assays permit to analyze pathways activity (ER expression, mTOR activation, CDK4/6 activation). Moreover, ultradeep sequencing and circulating DNA could be useful to highlight lethal subclones (ESR1 and PTEN mutations). Targeted sequencing, whole exome sequencing and SNP arrays can assess DNA repair defects (BRCA1/2 mutations or HRD assay). And finally, whole exome sequencing and RNA-sequencing are becoming interesting for tracking immune system (PDL1 overexpression neoantigens, mutation load). Overall, significant progress has been made in understanding metastatic breast cancers. It is necessary to proceed with further studies to translate these recent evidences into a clinical application and to provide patients new therapeutic options.

\section{Conflict of interest statement}

Concetta Elisa Onesti and Cécile Vicier: none.

Fabrice André: advisory boards and research grants for Astra; advisory boards, research grants and lecture for Novartis; research grants for Pfizer.

\section{References}

[1] Hanahan D, Weinberg RA. The hallmarks of cancer. Cell 2000;100:57-70.

[2] Hanahan D, Weinberg RA. Hallmarks of cancer: the next generation. Cell 2011;144:646-74.

[3] Dancey JE, Bedard PL, Onetto N, Hudson TJ. The genetic basis for cancer treatment decisions. Cell 2012;148:409-20.

[4] Pleasance ED, Cheetham RK, Stephens PJ, McBride DJ, Humphray SJ, Greenman CD, et al. A comprehensive catalogue of somatic mutations from a human cancer genome. Nature 2010;463:191-6.

[5] Mardis ER. The translation of cancer genomics: time for a revolution in clinical cancer care. Genome Med 2014;6(3):22.

[6] André F, Bachelot T, Commo F, Campone M, Arnedos M, Dieras V, et al. Comparative genomic hybridisation array and DNA sequencing to direct treatment of metastatic breast cancer: a multicentre, prospective trial (SAFIR01/UNICANCER). Lancet Oncol 2014;15:267-74.

[7] Eisenstein M. Foundation medicine. Nat Biotechnol 2012;30:14.

[8] Sleijfer S, Bogaerts J, Siu LL. Designing transformative clinical trials in the cancer genome era. J Clin Oncol 2013;31:1834-41. 
[9] DeSantis C, Ma J, Bryan L, Jemal A. Breast cancer statistics, 2013. CA Cancer J Clin 2014;64(1):52-62.

[10] Swain SM, Baselga J, Kim SB, Ro J, Semiglazov V, Campone M, et al. Pertuzumab, trastuzumab, and docetaxel in HER2-positive metastatic breast cancer. N Engl J Med 2015;372:724-34.

[11] Verma S, Miles D, Gianni L, Krop IE, Welslau M, Baselga J, , et alEMILIA Study Group. Trastuzumab emtansine for HER2-positive advanced breast cancer. N Engl J Med 2012;367:1783-91.

[12] Stephens PJ, Tarpey PS, Davies H, Van Loo P, Greenman C, Wedge DC, et al. The landscape of cancer genes and mutationam processes in breast cancer. Nature 2012;486:400-4.

[13] Cancer Genome Atlas Network. Comprehensive molecular portraits of human breast tumours. Nature 2012;490:61-70.

[14] Gewinner C, Wang ZC, Richardson A, Teruya-Feldstein J, Etemadmoghadam D, Bowtell D, et al. Evidence that inositol polyphosphate 4-phosphatase type II is a tumor suppressor that inhibits PI3K signaling. Cancer Cell 2009;16:115-25.

[15] Turner N, Lambros MB, Horlings HM, Pearson A, Sharpe R, Natrajan R, et al. Integrative molecular profiling of triple negative breast cancers identifies amplicon drivers and potential therapeutic targets. Oncogene 2010;29: 2013-23.

[16] Andre F, Job B, Dessen P, Tordai A, Michiels S, Liedtke C, et al. Molecular characterization of breast cancer with high-resolution oligonucleotide comparative genomic hybridization array. Clin Cancer Res 2009;15:441-51.

[17] Stemke-Hale K, Gonzalez-Angulo AM, Lluch A, Neve RM, Kuo WL, Davies M, et al. An integrative genomic and proteomic analysis of PIK3CA, PTEN, and AKT mutations in breast cancer. Cancer Res 2008;68:6084-91.

[18] Shah PD, Moynahan ME, Modi S, Caravella BA, Datko FM, Zamora S, et al. Phase I trial: PI3K $\alpha$ inhibitor BYL719 plus aromatase inhibitor (AI) for patients with hormone receptor-positive (HR+) metastatic breast cancer (MBC). SABSC. 2014 [Publication Number: PD5-3], 1424.

[19] Turner N, Pearson A, Sharpe R, Lambros M, Geyer F, Lopez-Garcia MA, et al, FGFR1 amplification drives endocrine therapy resistance and is a therapeutic target in breast cancer. Cancer Res 2010;70:2085-94.

[20] Shiang CY, Qi Y, Wang B, Lazar V, Wang J, Fraser Symmans W, et al. Amplification of fibroblast growth factor receptor-1 in breast cancer and the effects of brivanib alaninate. Breast Cancer Res Treat 2010;123:747-55.

[21] André F, Cortés J. Rationale for targeting fibroblast growth factor receptor signaling in breast cancer. Breast Cancer Res Treat 2015;150:1-8.

[22] Arnedos M, Scott V, Job B, De La Cruz J, Commo F, Mathieu MC, et al. Array CGH and PIK3CA/AKT1 mutations to drive patients to specific targeted agents: a clinical experience in 108 patients with metastatic breast cancer. Eur J Cancer 2012;48:2293-9.

[23] Bose R, Kavuri SM, Searleman AC, Shen W, Shen D, Koboldt DC, et al. Activating HER2 mutations in HER2 gene amplification negative breast cancer. Cancer Discov 2013;3:224-37.

[24] Finn RS, Crown JP, Lang I, Boer K, Bondarenko IM, Kulyk SO, et al. The cyclindependent kinase $4 / 6$ inhibitor palbociclib in combination with letrozole versus letrozole alone as first-line treatment of oestrogen receptor-positive, HER2-negative, advanced breast cancer (PALOMA-1/TRIO-18): a randomised phase 2 study. Lancet Oncol 2015;16:25-35.

[25] Hortobagyi GN, Piccart-Gebhart MJ, Rugo HS, Burris HA, Campone M, Noguchi S, et al. Correlation of molecular alterations with efficacy of everolimus in hormone receptor-positive, HER2-negative breast cancer: results from BOLERO-2. J Clin Oncol 2013;31 [suppl; abstr LBA509].

[26] Baselga J, Campone M, Piccart M, Burris HA, Rugo HS, Sahmoud T, et al. Everolimus in postmenopausal hormone-receptor-positive advanced breast cancer. N Engl J Med 2012 Feb 9;366:520-9.

[27] Loi S, Haibe-Kains B, Majjaj S, Lallemand F, Durbecq V, Larsimont D, et al. PIK3CA associated with gene signature of low mTORC1 signalingand better outcomes in estrogen receptor-positive breast cancer. Proc Natl Acad Sci USA 2010;107:10208-13.

[28] Loi S, Michiels S, Baselga J, Bartlett JM, Singhal SK, Sabine VS, et al. PIK3CA genotype and a PIK3CA mutation-related gene signature and response to everolimus and letrozole in estrogen receptor positive breast cancer. PLoS One 2013;8:e53292.

[29] Bosco EE, Wang Y, Xu H, Zilfou JT, Knudsen KE, Aronow BJ, et al. The retinoblastoma tumor suppressor modifies the therapeutic response of breast cancer. J Clin Inv 2007;117:218-28.
[30] Gerlinger M, Rowan AJ, Horswell S, Larkin J, Endesfelder D, Gronroos E, et al. Intratumor heterogeneity and branched evolution revealed by multiregion sequencing. N Engl J Med 2012 Mar 8;366:883-92.

[31] Burrel RA, McGranahan N, Bartek J, Swanton C. The causes and consequences of genetic heterogeneity in cancer evolution. Nature 2013;501:338-45.

[32] Landau DA, Klement K, Ziller MJ, Boyle P, Fan J, Gu H, et al. Locally disordered methylation forms the basis of intratumor methylome variation in chronic lymphocytic leukemia. Cancer Cell 2014;26:813-25.

[33] André F, Mardis E, Salm M, Soria JC, Siu LL, Swanton C. Prioritizing targets for precision cancer medicine. Ann Oncol 2014;25:2295-303.

[34] Haffner MC, Mosbruger T, Esopi DM, Fedor H, Heaphy CM, Walker DA, et al. Tracking the clonal origin of lethal prostate cancer. J Clin Invest 2013;123: 4918-22.

[35] Criscitiello C, Andre F, Thompson AM, De Laurentiis M, Esposito A, Gelao L, et al. Biopsy confirmation of metastatic sites in breast cancer patients: clinical impact and future perspectives. Breast Cancer Res 2014;16:205.

[36] Murtaza M, Dawson SJ, Tsui DWY, Gale D, Forshew T, Piskorz AM, et al. Noninvasive analysis of acquired resistance to cancer therapy by sequencing of plasma DNA. Nature 2013;497:108-12.

[37] Arnedos M, Filleron T, Dieci MV, Adam J, Robbins PB, Loi S, et al. Genomic and immune characterization of metastatic breast cancer $(\mathrm{MBC})$ : an ancillary study of SAFIR01 \& MOSCATO trials. Ann Oncol 2014;25(Suppl 4):iv116-36.

[38] Toy W, Shen Y, Won H, Green B, Sakr RA, Will M, et al. ESR1 ligand binding domain mutations in hormone resistant breast cancer. Nat Genet 2013;45: $1439-45$.

[39] Robinson DR, Wu YM, Vats P, Su F, Lonigro RJ, Cao X, et al. Activating ESR1 mutations in hormone-resistant metastatic breast cancer. Nat Genet 2013;45: $1446-51$.

[40] Juric D, Castel P, Griffith M, Griffith OL, Won HH, Ellis H, et al. Convergent loss of PTEN leads to clinical resistance to $\mathrm{PI}(3) \mathrm{K} \alpha$ inhibitor. Nature 2015;518: 240-4.

[41] Miki Y, Swensen J, Shattuck-Eidens D, Futreal PA, Harshman K, Tavtigian S et al. A strong candidate for the breast and ovarian cancer susceptibility gene BRCA1. Science 1994;266:66-71.

[42] Wooster R, Bignell G, Lancaster J, Swift S, Seal S, Mangion J, et al. Identification of the breast cancer susceptibility gene BRCA2. Nature 1995;378:789-92.

[43] Domchek SM, Weber BL. Clinical management of BRCA1 and BRCA2 mutation carriers. Oncogene 2006;25:5825-31.

[44] Farmer H, McCabe N, Lord CJ, Tutt AN, Johnson DA, Richardson TB, et al Targeting the DNA repair defect in BRCA mutant cells as a therapeutic strategy. Nature 2005;434:917-21.

[45] Tutt A, Robson M, Gaber JE, Domchek SM, Audeh MW, Weitzel JN, et al. Ora poly(ADP-ribose) polymerase inhibitor olaparib in patients with BRCA1 or BRCA2 mutations and advanced breast cancer: a proof-of-concept trial. Lancet 2010;376:235-44.

[46] McNeish I, Coleman R, Oza AM, Konecny GE, O'Malley D, Kichenadasse G, et al Preliminary results of ARIEL2, a phase 2 open-label study to identify ovarian cancer patients likely to respond to rucaparib. Ann Oncol 2014;25(Suppl 4): iv305-26.

[47] Alexandrov LB, Nik-Zainal S, Wedge DC, Aparicio SA, Behjati S, Biankin AV et al. Signatures of mutational processes in human cancer. Nature 2013;500: $415-21$.

[48] Ku GY, Yuan J, Page DB, Schroeder SE, Panageas KS, Carvajal RD, et al. Singleinstitution experience with ipilimumab in advanced melanoma patients in the compassionate use s setting: lymphocyte count after 2 doses correlates with survival. Cancer 2010;116:1767-75.

[49] Ji RR, Chasalow SD, Wang L, Hamid O, Schmidt H, Cogswell J, et al. An immune-active tumor microenvironment favors clinical response to ipilimumab. Cancer Immunol Immunother 2012;61:1019-31.

[50] Cha E, Klinger M, Hou Y, Cummings C, Ribas A, Faham M, et al. Improved survival with $\mathrm{T}$ cell clonotype stability after anti-CTLA-4 treatment in cancer patients. Sci Transl Med 2014;6:238ra70.

[51] Snyder A, Makarov V, Merghoub T, Yuan J, Zaretsky JM, Desrichard A, et al Genetic basis for clinical response to CTLA-4 blockade in melanoma. N Eng J Med 2014;371:2189-99.

[52] Apetoh L, Ghirindelli F, Tesniere A, Obeid M, Ortiz C, Criollo A, et al. Toll-like receptor 4-dependent contribution of the immune system to anticancer chemotherapy and radiotherapy. Nat Med 2007;13:1050-9. 